\title{
ON THE IDEMPOTENCY OF SOME COMPOSITE FUNCTORS
}

\author{
RAMÓN J. FLORES
}

\begin{abstract}
We present examples of localization functors whose composition with certain cellularization functors is not idempotent, and vice versa.
\end{abstract}

\section{INTRODUCTION}

The main goal of this note is to discuss some interesting questions raised by E. DrorFarjoun, concerning the effect of the successive application of localization and cellularization functors (see definitions in next section). We deal with Conjectures 3 and 7 of [Mis08, pages 62-63], which essentially ask for any localization functor $L$ and cellularization $C W$, which are homotopy idempotent by definition, whether the composite functors $L C W$ and $C W L$ are also homotopy idempotent. Note that the functors $L$ and $C W$ are respectively coaugmented and augmented, but in general neither $L C W$ nor $C W L$ admit coaugmentation nor augmentation.

In light of these considerations we use a previous description [FS07, Corollary 5.8] of the $B \mathbb{Z} / 2$-cellularization of the smallest in the family of Suzuki simple groups, $S z(8)$, to provide counterexamples to the questions of Farjoun. The following theorem summarizes our main results, which appear in the text as Proposition 3.3 and Proposition 3.5 ,

Theorem 1.1. If $C W$ represents cellularization with regard to a wedge $B \mathbb{Z} / 2 \vee$ $\bigvee_{p \text { odd prime }} M(\mathbb{Z} / p, 2)$ and $L$ denotes an $n$-Postnikov section for an appropriate choice of $n$, then the functors $L C W$ and $C W L$ are not homotopy idempotent.

The paper is structured as follows: next section is devoted to recall some notions of (co)Localization Theory, and also to a little comprehensive introduction of the problem of the idempotency of the functors $L C W$ and $C W L$; Section 3 contains our main results and their proofs.

Date: May 20, 2018.

The author is supported by MEC grant MTM2004-06686, MEC, Spain. 
Acknowledgments. We warmly thank Carles Broto, Richard Foote and Jérôme Scherer for their careful reading of the paper and their valuable comments.

\section{Preliminaries}

Recall that given a pointed map $f: A \rightarrow B$, a space $X$ is said to be $f$-local if the following conditions hold: the induced function $\operatorname{map}_{*}(B, X) \rightarrow \operatorname{map}_{*}(A, X)$ is a weak equivalence; the localization associated to $f$ is a coaugmented and idempotent endofunctor $L$ of the category of spaces; and every map $X \rightarrow Y$ from a space $X$ to a $f$-local space $Y$ factors through $L X$ in an unique way, up to homotopy. Analogously, given spaces $A$ and $X, X$ is said $A$-cellular if it is homotopy equivalent to an iterated homotopy colimit of copies of $A$. The $A$-cellularization of $X$, denoted by $C W_{A}$, is defined as an augmented idempotent endofunctor of the category of spaces such that the augmentation $C W_{A} X \rightarrow X$ is initial among all maps $f: Y \rightarrow X$ which induce a weak equivalence $\operatorname{map}_{*}(A, Y) \rightarrow \operatorname{map}_{*}(A, X)$. A comprehensive introduction to these functors can be found in the first two chapters of [Far95].

It is quite easy to find pairs of functors $C W$ and $L$ such that $C W L$ or $L C W$ are idempotent. For example, if $L$ is a Postnikov section $P[n]$, or more generally localization with regard the constant map $A \rightarrow *$ for a certain space $A$ (usually called $A$-nullification), and $C W$ is the $n$-connected cover functor (respectively $C W_{A}$ ), both composites are trivial. A similar phenomenon occurs if we apply $C W_{M(\mathbb{Z} / p, 2)}$, where $p$ is a prime, and then take the Bousfield-Kan $q$-completion at another prime $q$.

It can also happen that the composite of $L$ and $C W$ behaves like the identity functor (up to homotopy) over certain spaces, which immediately implies idempotency. An easy example is given by taking $L$ as the $R$-completion, for $R=\mathbb{Q}$ or $R=\mathbb{Z} / p$, of a simply-connected space, and $C W$ as the universal cover functor; then $L C W$ is the identity over $L X$, and $C W L$ is idempotent over $X$. So the problem is to find functors $L$ and $C W$ such that their combined effect is not so drastic as to kill the entire space, nor so weak that the composition gives back the original space. As one can expect, for "nice" spaces homological localization functors [Bou94] combined with cellularization with regard to $p$-primary meaningful spaces (as $B \mathbb{Z} / p$ or Moore spaces for $\mathbb{Z} / p$ ) have a good behavior in this context, and idempotency can be expected at least when working with nilpotent spaces. The same happens for some well-known nullification functors ([Bou94], [Bou97]). 
So, when searching for counterexamples there are two different but complementary paths: to seek for exotic localizations/cellularizations, or to apply the aforementioned functors to non-nilpotent spaces. The first strategy seems rather unpromising, as precise descriptions of the effect of $L$, or even more so of $C W$, are in general not available for functors that are outside the context of $p$-primary homotopy; and even less is known of the structure of $L C W X$ or $C W L X$. If the second path is taken, one quickly realizes that two features of $L$ and $C W$ are needed: their effect on the target space $X$ should be very precisely known, and both must change $X$ in a "moderately opposite" way, so $C W L X$ or $L C W X$ should be non-trivial but not very complicated spaces. This is the approach we take in next section, where our main results are proven.

\section{IDEMPOTENCY AND CLASSIFYING SPACES}

We will always consider cellularization with regard to the classifying space $B \mathbb{Z} / 2$, and we will denote this functor simply by $C W$. In turn, if $M$ is the wedge of all Moore spaces $M(\mathbb{Z} / q, 2)$ for $q$ odd, $L$ will be nullification with regard to a wedge of $M$ with a sphere $S^{n+1}$ whose dimension will be determined in due course.

To keep this exposition brief we refer the reader to [Gor80, 16.4] for details on the 2-local structure of $S z(8)$. As this group is our main object of study, we henceforth denote it simply by $G$.

Before applying the functors, we need some technical remarks concerning to the primary structure of $B G$. Bousfield-Kan $q$-completion of a space $X$ (see [BK72] for a main reference) is denoted by $X_{q}^{\wedge}$.

Lemma 3.1. The space $B G_{q}^{\wedge}$ is 2-connected for $q$ odd.

Proof. As $G$ is a simple group, the $q$-completion of $B G$ is simply-connected, and according to [BK72, VII.4.3], $\pi_{2} B G_{q}^{\wedge}$ must be a finite $q$-group for every $q$. Moreover, the Schur multiplier of $G$ is isomorphic to $\mathbb{Z} / 2 \times \mathbb{Z} / 2$, so $H_{2}\left(B G ; \mathbb{F}_{q}\right)=0$ by universal coefficients. By Hurewicz's Theorem, we have $\pi_{2} B G_{q}^{\wedge}=H_{2}\left(B G_{q}^{\wedge} ; \mathbb{F}_{q}\right)$, which is in turn isomorphic to $H_{2}\left(B G ; \mathbb{F}_{q}\right)$ because $B G$ is $q$-good [BK72, VII.5]. This completes the proof. 
Note that according to [RS01, Theorem 6.2], the space $B G_{q}^{\wedge}$ should be $M(\mathbb{Z} / q, 2)$ cellular for every $q$ odd, and then it is killed by $M(\mathbb{Z} / q, 2)$-nullification, i.e., $\mathbf{P}_{M(\mathbb{Z} / q, 2)} B G_{q}^{\wedge}$ is contractible.

Recall now from [Gor80] that a Sylow 2-subgroup, $S$, of $G$ satisfies: $Z(S)=S^{\prime}=$ $\Omega_{1}(S) \cong(\mathbb{Z} / 2)^{3}$ and $S / Z(S) \cong(\mathbb{Z} / 2)^{3}$. Thus $Z(S)=C l(S)$ is the minimal strongly closed subgroup that contains all elements of order 2 in $S$. The normalizer, $N$, of $S$ in $G$ acts transitively on the nonidentity elements of both $Z(S)$ and $S / Z(S)$. Following the notation of [FS07], let $\Gamma=N / Z(S) \cong(\mathbb{Z} / 2)^{3} \rtimes \mathbb{Z} / 7$. The following result describes the low-dimensional homology of $\Gamma$ :

Lemma 3.2. The group $\Gamma$ is 2-superperfect, i.e. $H_{i}(\Gamma, \mathbb{Z} / 2)=0$ for $i=1,2$.

Proof. The group $\Gamma$ is 2-perfect by [FS07, page 51], and moreover it is not hard to check that $H_{1}(\Gamma ; \mathbb{Z})=\mathbb{Z} / 7$. Using now Gaschütz's Theorem and the Fitting Lemma Gor80], it can also be seen that the Schur multiplier of $\Gamma$ is trivial. Then $H_{2}\left(\Gamma ; \mathbb{F}_{2}\right)$ is likewise trivial by universal coefficients, and we are done.

We can now give the precise definition of $L$. The group $\Gamma$ is 2-perfect and moreover has torsion in odd primes, so according to [Lev95, 1.1.4] $B \Gamma_{2}^{\wedge}$ has an infinite number of nonzero homotopy groups. If $n$ is the smallest natural number such that $\pi_{n} B \Gamma_{2}^{\wedge}$ is nontrivial, then our localization $L$ will be nullification with regard to the wedge $S^{n+1} \vee M$, where $M$ is the wedge of Moore spaces defined above. Observe that according to the previous lemma and $3.1, n>2$.

We are ready to explore the behavior of $B G$ under the recursive action of the functors $L$ and $C W$. As $n>1$, it is clear that $B G$ is $L$-local, and therefore by [FS07, Corollary 5.8], $C W B G \simeq C W L B G$ fits in a fibre sequence:

$$
C W B G \longrightarrow B G \longrightarrow B \Gamma_{2}^{\wedge} \times \prod_{q \neq 2} B G_{q}^{\wedge}
$$

where the product on the right is extended to odd primes. Here the map on the right is given by the composition

$$
B G \longrightarrow B G_{2}^{\wedge} \longrightarrow B N_{2}^{\wedge} \longrightarrow B \Gamma_{2}^{\wedge}
$$

where the second map is an equivalence because the normalizer of $S$ controls $G$-fusion in $S$ (see details in [FS07, Example 5.2]). 
Now let us describe $L C W B G$. Looping the base of the previous fibre sequencee we obtain another one, which is principal:

$$
\Omega\left(B \Gamma_{2}^{\wedge}\right) \times \Omega\left(\prod_{q \neq 2} B G_{q}^{\wedge}\right) \longrightarrow C W B G \longrightarrow B G .
$$

The space $B G$ is clearly $S^{n+1} \vee M$-null, so by [Far95, Proposition 3.D.3] the fibration is preserved by $L$. On the other hand, Lemma 3.1 implies that $\Omega\left(\prod_{q \neq 2} B G_{q}^{\wedge}\right)$ is simply connected, so it is $M$-cellular by [RS01, Theorem 6.2] and its $L$-localization is contractible. Then, by definition of $L$ we have that $L \Omega\left(B \Gamma_{2}^{\wedge}\right)$ has the homotopy type of an Eilenberg-Mac Lane space $K(H, n-1)$, where $H=\pi_{n} B \Gamma_{2}^{\wedge}$. Thus $L C W B G(=L C W L B G)$ is homotopy equivalent to a Postnikov piece $X$ whose only nonzero homotopy groups are $\pi_{1} X=G$ and $\pi_{n-1} X=H$.

We are now ready to give a solution (in the negative) to the first part of Problem 3 and Problem 7 in [Mis08, pages. 62-63].

Proposition 3.3. The functor $C W L$ is not idempotent.

Proof. Consider a non-trivial map $\sum^{n-2} B \mathbb{Z} / 2 \rightarrow K(H, n-1)$, which exists because $H$ is a finite 2-group, and observe that it remains essential when composing with the covering map $K(H, n-1) \rightarrow X$ (otherwise it would lift non-trivially to the discrete space $\Omega B G \simeq G$ ). On the other hand, as $B G$ is aspherical and $n>2$, there are no non-trivial maps from $\Sigma^{n-2} B \mathbb{Z} / 2$ to $B G$. Hence, $\operatorname{map}_{*}(B \mathbb{Z} / 2, B G)$ is not equivalent to $\operatorname{map}_{*}(B \mathbb{Z} / 2, X)$, and so $C W X \not C W B G$. As $X=L C W L B G$ and $B G \simeq L B G$, we are done.

So our goal now is to check that $L C W$ is not idempotent either. To undertake this task, we will need to explicitly compute $C W X$. A theorem of Chachólski [Cha96, 20.10] establishes that the $A$-cellularization of any space $Y$ is the fibre of the $\Sigma A$ nullification of the cofibre of the map $\bigvee_{[A, Y]_{*}} A \rightarrow Y$, where the wedge is extended to all the homotopy classes of maps $A \rightarrow Y$. In our case then we consider the cofibre sequence

$$
\bigvee_{[B \mathbb{Z} / 2, X]_{*}} B \mathbb{Z} / 2 \longrightarrow X \longrightarrow C
$$

and we need to describe its $\Sigma B \mathbb{Z} / 2$-nullification.

To accomplish this we first show that $C$ is simply connected. Because $G$ is generated by elements of order two (it is simple), by the Mayer-Vietoris sequence this amounts to checking that every element of order two in $\pi_{1} X=G$ is in the image of the 
homomorphism induced at the level of fundamental groups by a certain map $B \mathbb{Z} / 2 \rightarrow$ $X$. So, let $f$ be such a map, and $x$ be an element of $G$ of order two which is in the image of $\pi_{1} f$. As the fibre sequence 3.1 is principal and $S^{n+1} \vee M$ is equivalent to a suspension, the fibration $K(H, n-1) \rightarrow X \rightarrow B G$ which defines $X$ is principal by [DF09, Theorem 3.1]. Moreover, it is classified by the cohomology class defined by the composition $B G \rightarrow B \Gamma_{2}^{\wedge} \rightarrow K(H, n)$, where the second map is $(n+1)$-Postnikov section. According to the definition of $\Gamma$, the composition $B \mathbb{Z} / 2 \rightarrow B G \rightarrow K(H, n)$ is null-homotopic for every map $B \mathbb{Z} / 2 \rightarrow B G$. This implies in particular that $f$ lifts to $X$, so $C$ is simply connected.

Now we can describe $C$ more precisely: As $\bigvee B \mathbb{Z} / 2$ and $X$ are rationally trivial, $C$ is too by Mayer-Vietoris sequence in rational homology. Moreover, as the universal covering fibration of $X$ is principal, it is preserved by $p$-completion ([BK72, II.5.2]), and hence the "formal completions" $\left(\prod_{p} X_{p}^{\wedge}\right)_{\mathbb{Q}}$ and $\left(\prod_{p} C_{p}^{\wedge}\right)_{\mathbb{Q}}$ are also trivial. Now, Sullivan's arithmetic square [BK72, VI.8.1] implies that the cofibre splits as a product $\prod C_{q}^{\wedge}$ over all primes $q$. Clearly $\operatorname{map}_{*}\left(\Sigma B \mathbb{Z} / 2, C_{q}^{\wedge}\right)$ is weakly trivial for every $q$ odd; moreover, as $\bigvee_{[B \mathbb{Z} / 2, X]_{*}} B \mathbb{Z} / 2$ is $q$-acyclic, the $q$-completion of the cofibre sequence that defines $C$ gives an equivalence $B G_{q}^{\wedge} \simeq C_{q}^{\wedge}$. Then by Chachólski's Theorem $C W X$ is equivalent to the homotopy fibre of $X \rightarrow \mathbf{P}_{\Sigma B \mathbb{Z} / 2}\left(C_{2}^{\wedge}\right) \times \prod_{q \neq 2} B G_{q}^{\wedge}$, and we only need to describe $\mathbf{P}_{\Sigma B \mathbb{Z} / 2}\left(C_{2}^{\wedge}\right)$ to get a complete characterization of $C W X$.

Proposition 3.4. The space $\mathbf{P}_{\Sigma B \mathbb{Z} / 2}\left(C_{2}^{\wedge}\right)$ has the homotopy type of $B \Gamma_{2}^{\wedge}$.

Proof. We follow the same ideas as in the proof of [FS07, Proposition 5.5], see also [FF09, Section 3] for a comprehensive introduction to Zabrodsky Lemma in this context. We denote the $\Sigma B \mathbb{Z} / 2$-null space $\mathbf{P}_{\Sigma B \mathbb{Z} / 2}\left(C_{2}^{\wedge}\right)$ by $P$. We need to define maps between $P$ and $B \Gamma_{2}^{\wedge}$; and we start by constructing the map $P \rightarrow B \Gamma_{2}^{\wedge}$ by 
considering the diagram

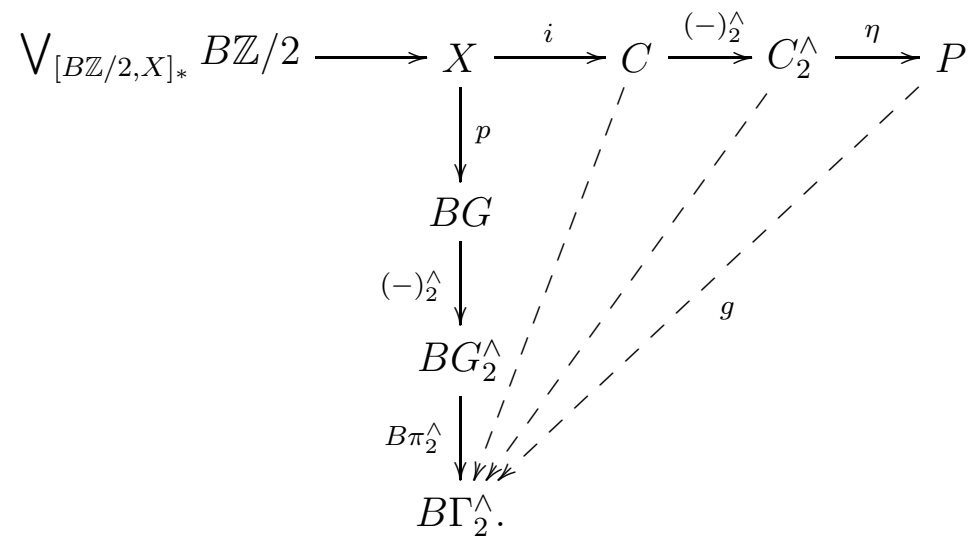

Here $\eta$ denotes the coaugmentation of the $\Sigma B \mathbb{Z} / 2$-nullification, and $B \pi_{2}^{\wedge}$ denotes the composition of the isomorphism $B G_{2}^{\wedge} \simeq B N_{2}^{\wedge}$ with the map induced in 2-completed classifying spaces by the projection $N \rightarrow \Gamma$. (Recall that $N$ stands for the normalizer of the Sylow 2-subgroup $S$ of $G$, which controls fusion in $S$.)

Observe that the composite $B \mathbb{Z} / 2 \rightarrow X \rightarrow B G$ is trivial for every map $B \mathbb{Z} / 2 \rightarrow X$. Therefore, the composite of the vertical maps extends to $C$ (all the extensions are considered up to homotopy). As $B \Gamma_{2}^{\wedge}$ is $\Sigma B \mathbb{Z} / 2$-null (see [Mil84, 9.9]) and 2-complete the corresponding map $C \rightarrow B \Gamma_{2}^{\wedge}$ also extends to $C_{2}^{\wedge}$ and then to $P$. So we have defined $g$, one of the desired maps.

Again for clarity, we summarize the information of the second part of the proof in a new diagram, which we prove to be commutative up to unpointed homotopy:

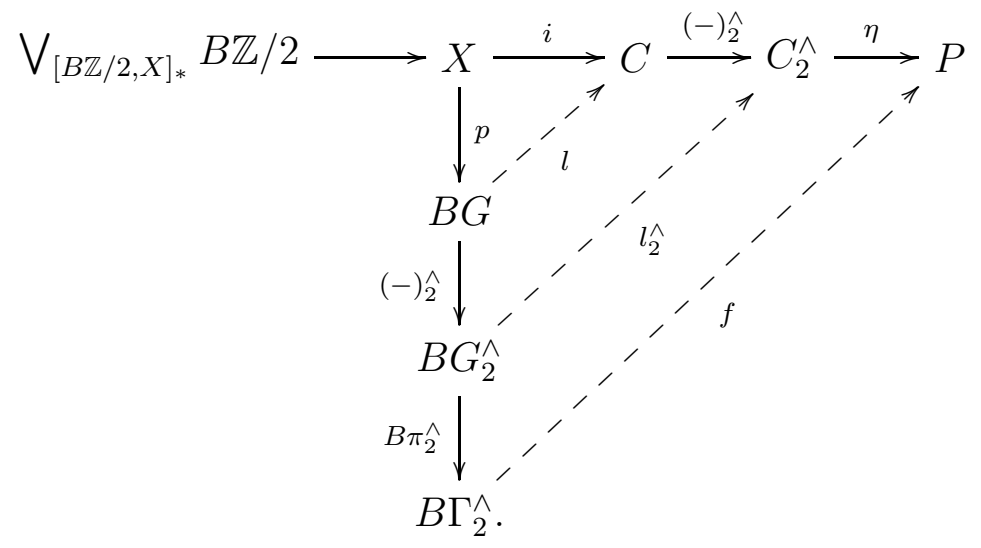

To define the second map we consider the covering fibre sequence:

$$
K(H, n-1) \longrightarrow X \longrightarrow B G
$$




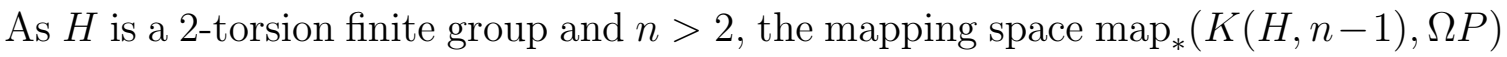
is weakly trivial. But the composition $K(H, n-1) \rightarrow X \rightarrow C \rightarrow P$ is inessential, so Dwyer's version of the Zabrodsky Lemma ([Dwy96, Proposition 3.5], see also [CCS, Lemma 2.3]) establishes the existence of an extension (up to unpointed homotopy)

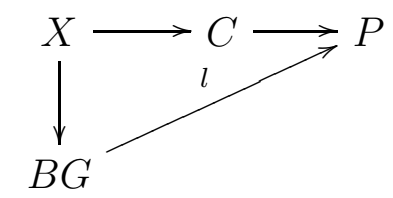

which, in particular, is null-homotopic if and only if the upper composition is. As $P$ is 2-complete, the extension $B G \rightarrow P$ factors through $B G_{2}^{\wedge}$, which has the homotopy type of $B N_{2}^{\wedge}$. Now for $Z(S)=C l(S)<S$ as discussed just before Lemma 3.2, there is another fibre sequence $B C l(S) \rightarrow B N \rightarrow B \Gamma$. By the definition of $\Gamma$ and $P$, the composition

$$
B C l(S) \longrightarrow B N \longrightarrow B N_{2}^{\wedge} \simeq B G_{2}^{\wedge} \longrightarrow P
$$

is trivial. Then as $P$ is $\Sigma B \mathbb{Z} / 2$-null, Zabrodsky's Lemma again shows the existence of an extension from $B N_{2}^{\wedge} \rightarrow P$ to $B \Gamma_{2}^{\wedge}$. This is the other map we needed, and we denote it by $f$.

Our task now is to establish that $f \circ g$ and $g \simeq f$ are (pointed) homotopic to the respective identities. As $P$ and $B \Gamma_{2}^{\wedge}$ are simply connected, it is enough to check this in the unpointed category. Let us denote by $F$ the composition of the horizontal maps in the previous diagram, and by $G$ the composition of the vertical maps.

Consider first the composition $f \circ g: P \rightarrow P$. The universal property of localization guarantees that $f \circ g \simeq I d_{P}$ if and only if $f \circ g \circ \eta \simeq \eta$. Moreover, as $P$ is 2-complete, this is equivalent to establishing that $f \circ g \circ \eta \circ(-)_{2}^{\wedge} \simeq \eta \circ(-)_{2}^{\wedge}$. Now the Puppe sequence of the cofibration $\bigvee_{[B \mathbb{Z} / 2, X]_{*}} B \mathbb{Z} / 2 \rightarrow X$ implies that the latter is true if and only if the maps are homotopic when precomposing with $i$. By the definition of $F$, this is the same as checking that $f \circ g \circ F$ is homotopic to $F$. According to Diagram 1.1, $g \circ F \simeq G$, and then Diagram 1.2 implies $f \circ G \simeq F$.

On the other hand, consider $g \circ f: B \Gamma_{2}^{\wedge} \rightarrow B \Gamma_{2}^{\wedge}$. As this space is 2-complete and $\Sigma B Z / 2$-null, Zabrodsky's Lemma again applied to the fibration $B C l(S) \rightarrow B G \rightarrow B \Gamma$ implies that $g \circ f \simeq I d_{B \Gamma_{2}^{\wedge}}$ if and only if $g \circ f \circ B \pi_{2}^{\wedge} \circ(-)_{2}^{\wedge}$ is homotopic to $B \pi_{2}^{\wedge} \circ(-)_{2}^{\wedge}$. Applying the Zabrodsky Lemma again to the universal covering fibration of $X$, we obtain that the latter is equivalent to $g \circ f \circ B \pi_{2}^{\wedge} \circ(-)_{2}^{\wedge} \circ p \simeq(-)_{2}^{\wedge} \circ p$, or, in other 
words, $g \circ f \circ G \simeq G$. By Diagram 1.2 now, $f \circ G \simeq F$, and then by Diagram 1.1, $g \circ F \simeq G$. So we are done.

Proposition 3.5. The functor LCW is not homotopy idempotent.

Proof. The previous proposition gives a fibre sequence

$$
C W X \longrightarrow X \longrightarrow B \Gamma_{2}^{\wedge} \times \prod_{q \text { odd }} B G_{q}^{\wedge}
$$

that characterizes the cellularization of $X$. Recall at this point that $X$ is, by definition, $L C W L B G \simeq L C W B G$. We show how this space and the previous description of $C W X$ give a counterexample for the second part of Problem 7 of Farjoun in [Mis08].

As the map $X \rightarrow B \Gamma_{2}^{\wedge} \times \prod_{q \text { odd }} B G_{q}^{\wedge}$ factors through $B G$, its composition with $K(H, n-1) \rightarrow X$ is homotopically trivial, and thus the long exact homotopy sequence of the previous fibration defines $\pi_{n-1} C W X$ as an extension

$$
\pi_{n}\left(B \Gamma_{2}^{\wedge} \times \prod_{q \text { odd }} B G_{q}^{\wedge}\right) \longrightarrow \pi_{n-1} C W X \longrightarrow H
$$

Observe that $L$ does not affect the 2-torsion subgroup of $\pi_{n-1} C W X$, which is therefore isomorphic to the 2-torsion subgroup of $\pi_{n-1} L C W X$. In particular it is not isomorphic to the 2-torsion subgroup of $\pi_{n-1} X=H$ because $\pi_{n}\left(B \Gamma_{2}^{\wedge} \times \prod_{q \text { odd }} B G_{q}^{\wedge}\right)$, by definition of $n$, is nontrivial. Hence $L C W B G$ is not homotopy equivalent to $L C W X$, which is by definition $L C W L C W B G$. So $L C W$ is not homotopy idempotent either, and the argument is complete.

\section{REFERENCES}

[Bou94] A.K. Bousfield. The localization of spaces with respect to homology. Topology, 14: 133-150, 1975.

[Bou94] A.K. Bousfield. Localization and periodicity in unstable homotopy theory. J. Amer. Math. Soc., 7(4): 831-873, 1994.

[Bou97] A.K. Bousfield. Homotopical localization of spaces. Amer. J. Math., 119(6): 13211354, 1997.

[BK72] A. K. Bousfield and D. M. Kan. Homotopy limits, completions and localizations. Springer-Verlag, Berlin, 1972. Lecture Notes in Mathematics, Vol. 304.

[CCS] N. Castellana, J. A. Crespo, and J. Scherer. Postnikov pieces and BZ/ $/ p$-homotopy theory. Trans. Amer. Math. Soc., 359(4):1791-1816, 2007. 
[Cha96] W. Chachólski. On the functors $C W_{A}$ and $P_{A}$. Duke Math. J., 84(3):599-631, 1996.

[DF09] W.G. Dwyer and E.Dror-Farjoun Localization and cellularization of principal fibrations. , Preprint, available at http://www.nd.edu/ wgd/Dvi/LocalizationCellularizationPrincipalFibrations.pdf

[Dwy96] W. G. Dwyer. The centralizer decomposition of BG. In Algebraic topology: new trends in localization and periodicity (Sant Feliu de Guíxols, 1994), volume 136 of Progr. Math., pages 167-184. Birkhäuser, Basel, 1996.

[Far95] E. Dror-Farjoun. Cellular spaces, null spaces and homotopy localization, volume 1622 of Lecture Notes in Mathematics. Springer-Verlag, Berlin, 1996.

[FF09] R.J. Flores and R. Foote. The cellular structure of the classifying spaces of finite groups. Preprint, available at http://arxiv.org/pdf/0809.4116.

[FS07] R.J. Flores and J. Scherer. Cellularization of classifying spaces and fusion properties of finite groups. J. Lond. Math. Soc.(2) 76(1):41-56, 2007.

[Gor80] D. Gorenstein. Finite groups. Chelsea Publishing Co., New York, second edition, 1980.

[Lev95] R. Levi. On finite groups and homotopy theory. Mem. Amer. Math. Soc., 118(567):xiv+100, 1995.

[Mil84] H. Miller. The Sullivan conjecture on maps from classifying spaces. Ann. of Math. (2), 120(1):39-87, 1984.

[Mis08] Guido's book of conjectures. A gift to Guido Mislin on the occasion of his retirement from ETHZ, June 2006. Collected by Indira Chatterji.. Enseign. Math.,54(2): vols. $1-3,2008$.

[RS01] J. L. Rodríguez and J. Scherer. Cellular approximations using Moore spaces. In Cohomological methods in homotopy theory (Bellaterra, 1998), volume 196 of Progr. Math., pages 357-374. Birkhäuser, Basel, 2001.

Ramón J. Flores

Departamento de Estadística, Universidad Carlos III de Madrid, C/ Madrid 126

E - 28903 Getafe

e-mail: rflores@est-econ.uc3m.es 